\title{
Pressure Ulcer Scale for Healing (PUSH): confiabilidade interobservadores na avaliação de úlceras venosas*
}

Gabriela Rodrigues Alves ${ }^{1}$, Suelen Gomes Malaquias², Maria Márcia Bachion ${ }^{3}$

\section{RESUMO}

Objetivou-se avaliar a confiabilidade interobservadores da Pressure Ulcer Scale for Healing (PUSH), em pacientes com úlceras venosas. Trata-se de pesquisa metodológica, realizada em salas de curativos de unidades ambulatoriais de saúde, no período de junho de 2016 a agosto de 2017. Participaram 10 enfermeiras, que realizaram 46 observações em 35 lesões. Cada observação foi realizada por uma enfermeira de referência para a avaliação considerada padrão ouro e duas enfermeiras participantes da pesquisa como sujeito. Para análise de concordância, utilizou-se o índice Kappa e coeficiente de correlação intraclasse. Valores de Kappa das subescalas variaram de 0,6 a 0,85 ( $p<0,001)$. Houve concordância interobservador muito boa para "área" e "quantidade exsudato" e boa para "tipo de tecido" e "escore total". Os coeficientes de correlação intraclasse foram >0,9 o que indica excelente confiabilidade. Conclui-se que a PUSH apresenta confiabilidade interobservador satisfatória para avaliação de úlceras venosas.

Descritores: Cicatrização; Reprodutibilidade dos Testes; Úlcera Varicosa; Avaliação em Enfermagem.

\footnotetext{
* Este artigo foi elaborado de acordo com Guidelines for Reporting Reliability and Agreement Studies (GRRAS).

${ }^{1}$ Enfermeira, Mestre em Enfermagem. E-mail: gabrielarodriguesalves@gmail.com.

${ }^{2}$ Enfermeira, Doutora em Ciências da Saúde. Professora Adjunta da Faculdade de Enfermagem da Universidade Federal de Goiás. Goiânia, GO, Brasil. E-mail: sgmalaquias@gmail.com.

${ }^{3}$ Enfermeira, Doutora em Enfermagem. Professora Titular da Faculdade de Enfermagem da Universidade Federal de Goiás. Goiânia, GO, Brasil. Email: mbachion@gmail.com.
}

Artigo recebido: 20/09/2017.

Artigo aprovado: 28/03/2018.

Artigo publicado: 19/12/2018.

Como citar esse artigo:

Alves GR, Malaquias SG, Bachion MM. Pressure Ulcer Scale for Healing (PUSH): confiabilidade interobservadores na avaliação de úlceras venosas. Rev. Eletr. Enf. [Internet]. 2018 [acesso em: ];20:v20a33. Disponível em: https://doi.org/10.5216/ree.v20.49411. 


\section{INTRODUÇÃO}

As úlceras crônicas, em especial, de etiologia venosa, são muitas vezes avaliadas e tratadas de forma assistemática e inapropriada na prática clínica ${ }^{(1)}$. Agrava esse cenário a falta de um instrumento de avaliação que possa evidenciar os resultados do tratamento realizado.

Entre os parâmetros bem estabelecidos, que devem ser levados em conta na avaliação das condições de cicatrização, estão: tamanho, profundidade, condições de bordas e da pele peri-lesão, tipo de tecido e quantidade, exsudato, sinais de infecção e dor ${ }^{(2)}$.

Embora originariamente desenvolvida para avaliação de cicatrização de úlceras por pressão ${ }^{(3)}$, a Pressure Ulcer Scale for Healing $\left(\mathrm{PUSH}^{\circ}\right)$, considera alguns desses parâmetros e passou a ser utilizada na avaliação da cicatrização de outras feridas, crônicas ${ }^{(4-6)}$ e agudas ${ }^{(6)}$. Entre as vantagens deste instrumento, destacam-se a simplicidade e objetividade de seu uso, a partir da aplicação das subescalas "área da ferida", "quantidade de exsudato" e "tipo de tecido" (3,7).

A PUSH ${ }^{(3)}$ foi traduzida para a versão português do Brasil ${ }^{(4)}$ e testada a confiabilidade interobservadores da versão traduzida em pessoas com úlceras de perna, a qual apresentou características psicométricas satisfatórias ${ }^{(8)}$.

Considerando a importância de testar as propriedades psicométricas da PUSH em diferentes contextos e diferentes populações, contemplando a etiologia de úlceras crônicas mais prevalentes ${ }^{(9)}$, emerge o interesse no seu estudo no contexto da avaliação específica de úlceras venosas, com aplicação por enfermeiras que atuam na prática clínica.

Estas lesões destacam-se como as mais frequentes entre as úlceras de perna, e seu tratamento ocorre notadamente na rede ambulatorial da atenção básica em saúde ${ }^{(10)}$. Para sistematizar o cuidado com a pessoa com úlcera venosa, os enfermeiros devem avaliar a lesão ${ }^{(11)}$ e a aplicação de um instrumento de para descrever a evolução da cicatrização pode ser útil. Nesse contexto, a PUSH pode ser um instrumento promissor, pois mensura os principais parâmetros necessários na avaliação(12).

Contudo, tratando-se de lesão com características particulares, em relação ao aspecto do esfacelo, quantidade do exsudato e variação na área, quando comparadas com as úlceras por pressão, podem oferecer maior dificuldade para a avaliação pelos profissionais.

Dessa forma, este estudo teve como objetivo avaliar a confiabilidade interobservador da Pressure Ulcer Scale for Healing (PUSH) em pacientes com úlceras venosas.

\section{MÉTODOS}

Trata-se de estudo inserido no projeto matriz "Tradução, adaptação transcultural, confiabilidade e responsividade de escalas de avaliação da capacidade funcional, cicatrização e qualidade de vida de pessoas com úlceras venosas" aprovado no Comitê de Ética em Pesquisa da UFG (protocolo no 1.500.799), e conta com autorização do NPUAP para utilização da PUSH.

A coleta de dados foi realizada de abril de 2016 a agosto de 2017, em uma metrópole na região Centro-oeste do Brasil.

Para realizar no mínimo 30 observações, seguindo o padrão de estudos dessa natureza ${ }^{(8,13)}$, foram elegíveis para o estudo enfermeiros com seis meses a um ano de atuação no atendimento a pessoas com úlceras venosas de três a quatro vezes por semana, ou enfermeiros que atuavam nessa área há um ano ou mais, que realizassem esse atendimento uma a duas vezes por semana. Aqueles que aceitaram participar do estudo assinaram o Termo de Consentimento Livre e Esclarecido. Os enfermeiros foram treinados para o uso da escala, em um encontro com duração 
de 30 minutos em média, e na ocasião foi explicado o protocolo de avaliação (Apêndice 1) a ser utilizado pelos observadores na pesquisa.

Os critérios de inclusão dos usuários da rede de saúde como participantes da pesquisa foram: diagnóstico de úlcera venosa, estabelecido a partir da identificação de sinais clínicos específicos dessa etiologia (varizes, edema, hemossiderose, lipodermatoesclerose, entre outros), bem como resultado do índice tornozelo braço (ITB) entre 0,8 e 1,3. Excluíram-se aqueles que apresentaram sinais clínicos de comprometimento arterial moderado ou severo (palidez à elevação, pulsos diminuídos/ausentes, claudicação intermitente).

A PUSH inclui três parâmetros ou subescalas ${ }^{(3)}$ - área da ferida, cujos subescores variam de zero a 10; quantidade de exsudato, classificados em ausente, pequeno, moderado e grande, que correspondem, aos subescores de zero a três, respectivamente, e tipo de tecido no leito da ferida, que corresponde ao pior tecido presente no leito da lesão(7) que pode ser caracterizado em: ferida fechada, tecido epitelial, tecido de granulação, esfacelo e tecido necrótico, que são definidos por subescore de zero a quatro, respectivamente. A pontuação final é obtida mediante a soma dos escores dessas subescalas e varia de zero a 17, sendo que, quanto maior o escore total, piores são as condições de cicatrização da úlcera ${ }^{(7)}$.

Durante a realização dos curativos, a aplicação da escala ocorreu por três observadores, de forma simultânea e independente, sendo duas enfermeiras clínicas e uma enfermeira pesquisadora, com expertise na área; esta última foi tomada como referência para avaliação considerada como padrão ouro.

\section{Coleta de dados}

$\mathrm{Na}$ aplicação da escala, cada observador, avaliou no exame visual a quantidade de exsudato e registrou o subescore correspondente em seu instrumento privativo. Após a limpeza da ferida com SF 0,9\%, cada um avaliou o tipo de tecido presente no leito da lesão. Então, dois observadores retiraram-se do recinto e, um a um, em revezamento, procedeu à mensuração da largura e comprimento da lesão, por meio de régua de papel, estéril, graduada com precisão de milímetros. A seguir calcularam a área em $\mathrm{cm}^{2}$. Durante a mensuração da lesão, foi necessário recorrer a um gravador ou auxiliar de pesquisa para o registro das medidas, uma vez que os observadores estavam com luvas estéreis.

O NPUAP não define os parâmetros de avaliação da quantidade do exsudato, então, para esse estudo consideraram-se as características de umidade do curativo anterior, incluindo a extensão da área de impregnação do exsudato, em relação à dimensão do leito da lesão, e o grau de saturação da cobertura, em que: zero(0) = exsudato ausente (leito da ferida seco e sem umidade visível) ${ }^{(14)}$, um(1) = exsudato pequeno (saturação de 25 a $50 \%$ da cobertura usada no curativo, em área correspondente ao tamanho da lesão), dois(2) = exsudato moderado (saturação de 50 a $75 \%$ da cobertura usada no curativo, em área correspondente ao tamanho da lesão) e três(3) = exsudato grande (saturação maior que $75 \%$ da cobertura usada no curativo, em área maior que o leito da lesão $)^{(15)}$. Além disso, foi considerado o tempo de permanência da cobertura na lesão. Em síntese, foi levado em conta, extensão da área de impregnação do exsudato, grau de saturação e tempo de contato da cobertura com a lesão.

Em conformidade às instruções do NPUAP(3) para avaliar o tecido presente na lesão, considerou-se o pior tipo de tecido presente no leito e/ou bordas da lesão. Foram consideradas as características dos tecidos e classificados os escores em quatro(4) = tecido necrótico (escara): tecido de coloração preta, marrom ou castanha que adere firmemente ao leito ou as bordas da ferida e pode apresentar-se mais endurecido ou mais amolecido, do que a pele perilesional, três(3) = esfacelo: tecido de coloração amarela ou branca que adere ao leito da ferida e apresenta-se como cordões ou crostas grossas, podendo ainda ser mucinoso, dois(2) = tecido de granulação: tecido descoloração rósea ou vermelha, 
de aparência brilhante, úmida e granulosa, um(1) = tecido epitelial: para as feridas superficiais, aparece como um novo tecido róseo ou brilhante (pele) que se desenvolve a partir das bordas ou como “ilhas" na superfície da lesão, zero(0) = ferida fechada ou recoberta: a ferida está completamente coberta com epitélio (nova pele).

De acordo com o NPUAP, a medida da área se dá avaliando-se o maior comprimento e maior largura, no sentindo céfalo-caudal, no eixo longitudinal, por meio da utilização de uma régua graduada. Considerando que as úlceras venosas podem cicatrizar, passando de uma única lesão para duas, menores, ou ainda, duas lesões podem confluir para uma lesão maior (Figura 1), padronizou-se que, na presença de duas lesões próximas, com distância inferior a $2 \mathrm{~cm}$ entre elas, seria considerada lesão única ${ }^{(16)}$, realizando-se o registro das áreas de forma independente, somando-as, em seguida.

Figura 1: Úlceras venosas de perna (plano lateral), com distância inferior a $2 \mathrm{~cm}$. Lesão inferior com ilha de epitelização. Goiânia, GO, Brasil, 2017.

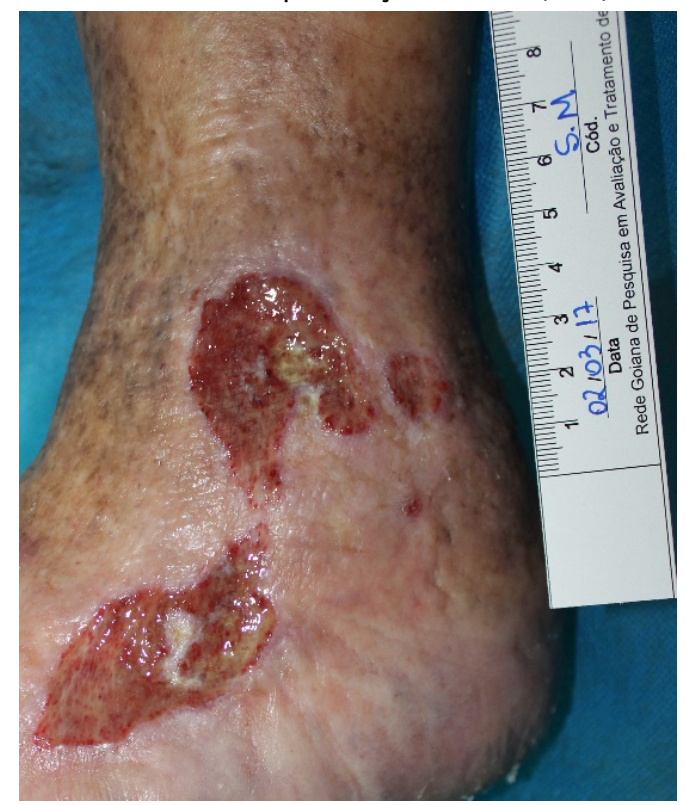

Fonte: Arquivo da Rede Goiana de Pesquisa em Avaliação e Tratamento de Feridas.

Ainda, em casos de ilhas de epitelização, buscaram-se as maiores extensões do leito da úlcera, sem sobrepor essas regiões. Nos casos de impossibilidade, realizou-se a mensuração da largura ou comprimento e descontou-se a área epitelizada dessa região.

\section{Análise de dados}

A análise dos dados foi realizada por meio do software Stata, versão 14.0 e os gráficos foram construídos no programa Statistica, versão 7.0. Foi utilizada estatística descritiva, medidas de tendência central, e, para o cálculo do índice de concordância, foi utilizado o índice kappa, considerando: $k<0,20=$ concordância pobre, 0,21 a 0,40 =

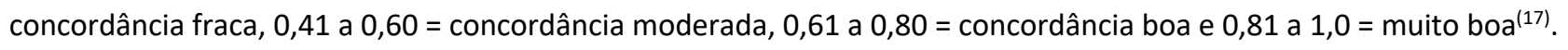

Utilizou-se ainda coeficiente de correlação intraclasse $(\mathrm{CCl})$, sendo, $\mathrm{CCl}$ inferior a 0,5 indicativo de confiabilidade pobre, 0,5 a 0,75, moderada, 0,75 a 0,90, boa e > 0,90 confiabilidade excelente ${ }^{(13)}$. 


\section{RESULTADOS}

Participaram do estudo 10 enfermeiras que trabalhavam em diferentes instituições de saúde, denominadas no contexto da presente investigação como enfermeiras clínicas, das quais $20 \%$ atuavam de seis meses a um ano, na área de tratamento de pessoas com úlceras venosas, 30\% de um a cinco anos e $50 \%$ de cinco anos ou mais.

Os três enfermeiros pesquisadores foram referência para a observação considerada como padrão ouro para a análise (Enf PO).

Foram analisadas 35 úlceras venosas, por trios de observadores (duas enfermeiras clínicas e uma enfermeira pesquisadora referência para a observação padrão ouro), em diferentes combinações, os quais realizaram quarenta e seis observações.

O tamanho das lesões que compuseram o estudo variou entre $0,02 \mathrm{~cm}^{2}$ e 551,0 cm², com média de $90,04 \mathrm{~cm}^{2}$ \pm 153 e mediana de $18,25 \mathrm{~cm}^{2}$.

A seguir são apresentados os resultados referentes aos subescores dos três parâmetros da PUSH e o escore total para as três observadoras.

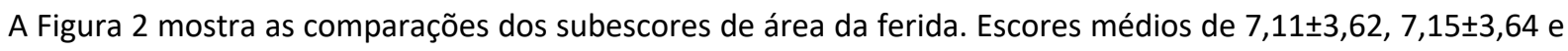
7,20 $\pm 3,53$ foram atribuídos, respectivamente, pelo enfermeiro de referência para a observação considerada padrão ouro (Enf PO), enfermeiro clínico um e (Enf 1) e dois (Enf 2).

Figura 2: Gráfico de médias e IC 95\% comparativo dos subescores da subescala área das úlceras venosas. Goiânia, GO, Brasil, 2017.

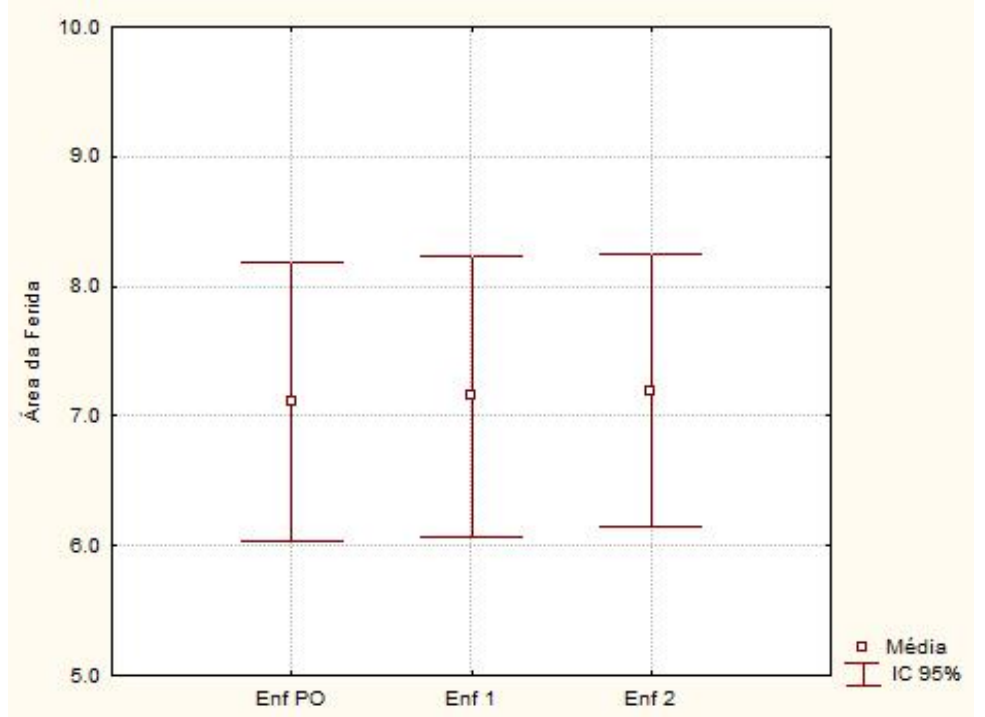

Legenda: Enf PO = enfermeira de referência para observação considerada padrão-ouro; Enf 1 = enfermeira clínica 1 ; Enf 2 = enfermeira clínica 2; Kappa Enf PO x Enf $1=0,881$ - $p<0,001$; Kappa Enf PO x Enf $2=0,853-p<0,001$.

A análise mostrou uma concordância entre o Enf PO e Enf 1 de 91,3\% ( $n=42$ ) e com o Enf 2 de 89,1\% ( $n=41)$ na avaliação da área das feridas e valores de $k$ acima de 0,80 , o que indica muito boa concordância(17) de interobservadores nesse item.

A Figura 3 mostra as comparações dos subescores da quantidade de exsudato. Foram encontrados escores

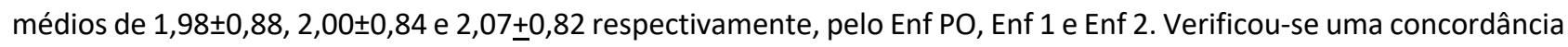
entre o Enf PO e Enf 1 de 89,1\% ( $n=41)$ e com o Enf 2 de 82,6\% ( $n=38$ ) na avaliação da quantidade de exsudato e valores de $\mathrm{K}$ que indicam concordância boa ou muito boa ${ }^{(17)}$ dos observadores para esse item. 
Figura 3: Gráfico de médias e IC $95 \%$ comparativo dos subescores da subescala quantidade de exsudato das úlceras venosas. Goiânia, GO, Brasil, 2017.

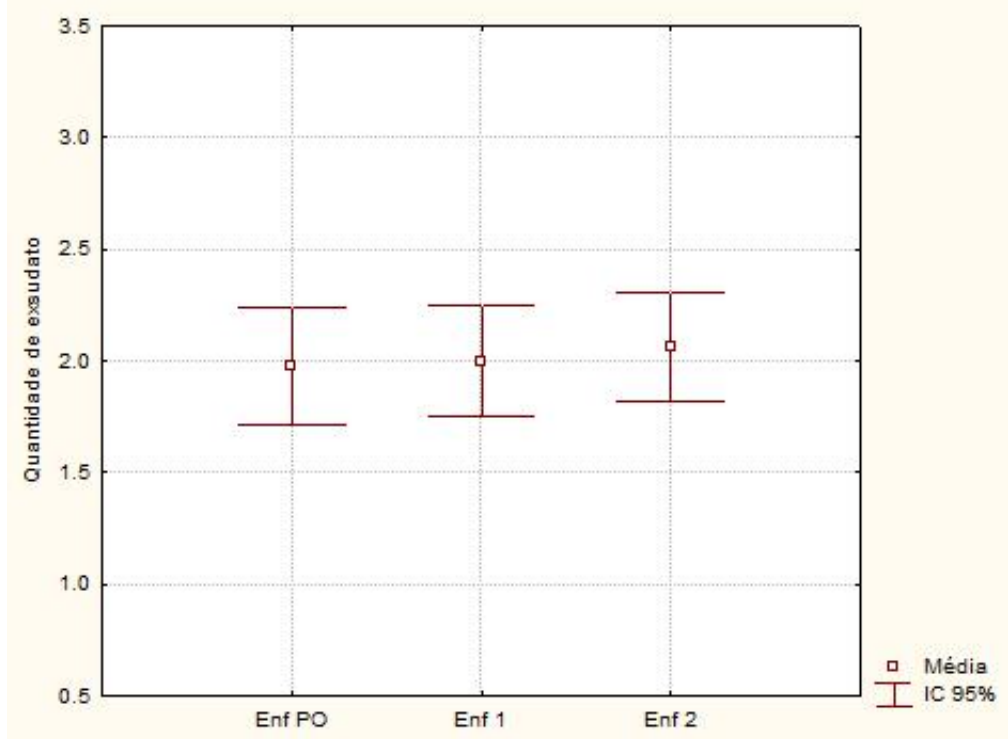

Legenda: Enf PO = enfermeira de referência para observação considerada padrão-ouro; Enf 1 = enfermeira clínica 1; Enf 2 = enfermeira clínica 2; Kappa Enf PO x Enf 1 = 0,835 - p 0,001; Kappa Enf PO x Enf $2=0,739-p<0,001$.

A Figura 4 apresenta as comparações dos subescores do tipo de tecido. Foram atribuídos escores médios de

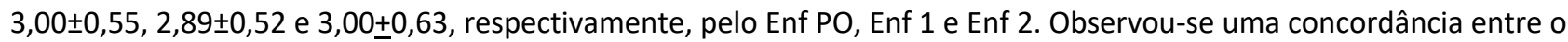
Enf PO e Enf 1 de 87,0\% ( $n=40)$ e com o Enf 2 de 89,1\% ( $n=41)$ na avaliação do tipo de tecido presente no leito das úlceras venosas.

Figura 4: Gráfico de médias e IC 95\% comparativo dos subescores da subescala tipo de tecido presente no leito das úlceras venosas. Goiânia, GO, Brasil, 2017.

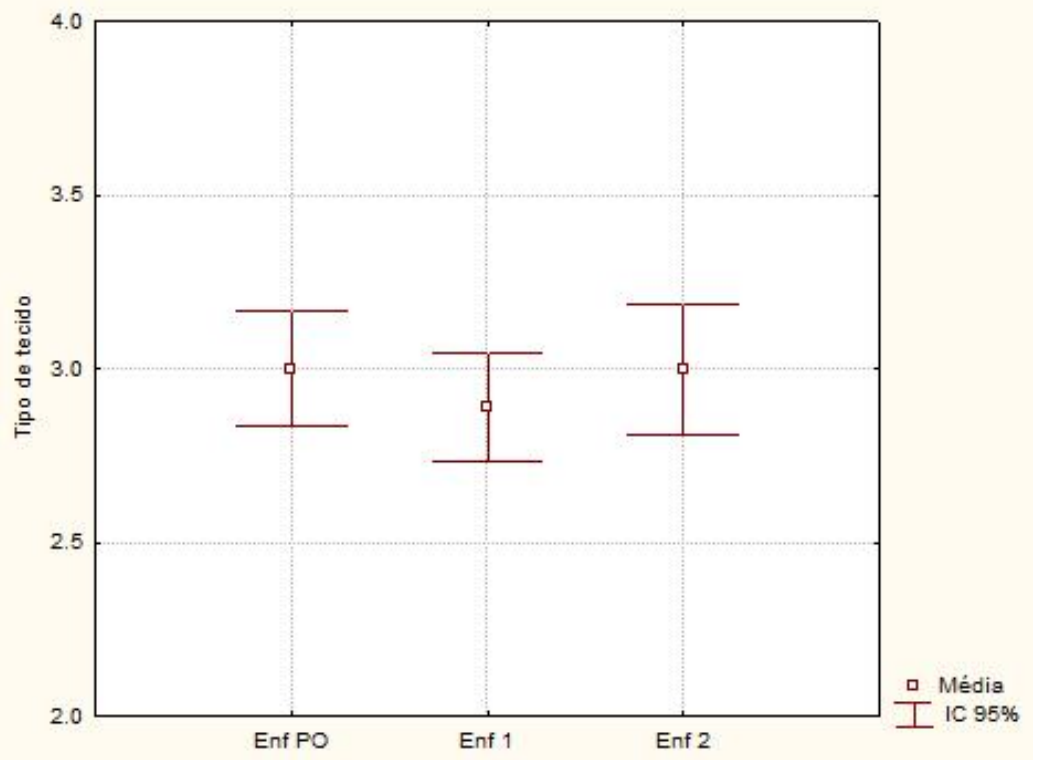

Legenda: Enf PO = enfermeira de referência para observação considerada padrão-ouro; Enf 1 = enfermeira clínica 1; Enf 2 = enfermeira clínica 2; Kappa Enf PO x Enf 1 = 0,715 - p<0,001; Kappa Enf PO x Enf $2=0,789-p<0,001$.

Os valores de $\mathrm{k}$ indicam boa concordância ${ }^{(17)}$ interobservadores na avaliação desse item.

A Figura 5 apresenta as comparações do escore total da PUSH. Foram atribuídos escores médios de 12,07士4,62, 12,02 $\pm 4,73$ e 12,24+4,57, respectivamente, pelo Enf PO, Enf 1 e Enf 2. Observou-se uma concordância entre o Enf PO e 
Enf 1 de 78,3\% ( $n=36$ ) e com o Enf 2 de 67,4\% ( $n=31$ ) na avaliação da cicatrização das úlceras venosas. Os valores de $\mathrm{k}$, indicam que houve boa concordância interobservadores ${ }^{(17)}$ na avaliação global.

Figura 5: Gráfico de médias e IC 95\% comparativo dos escores totais da PUSH na avaliação de úlceras venosas. Goiânia, GO, Brasil, 2017.

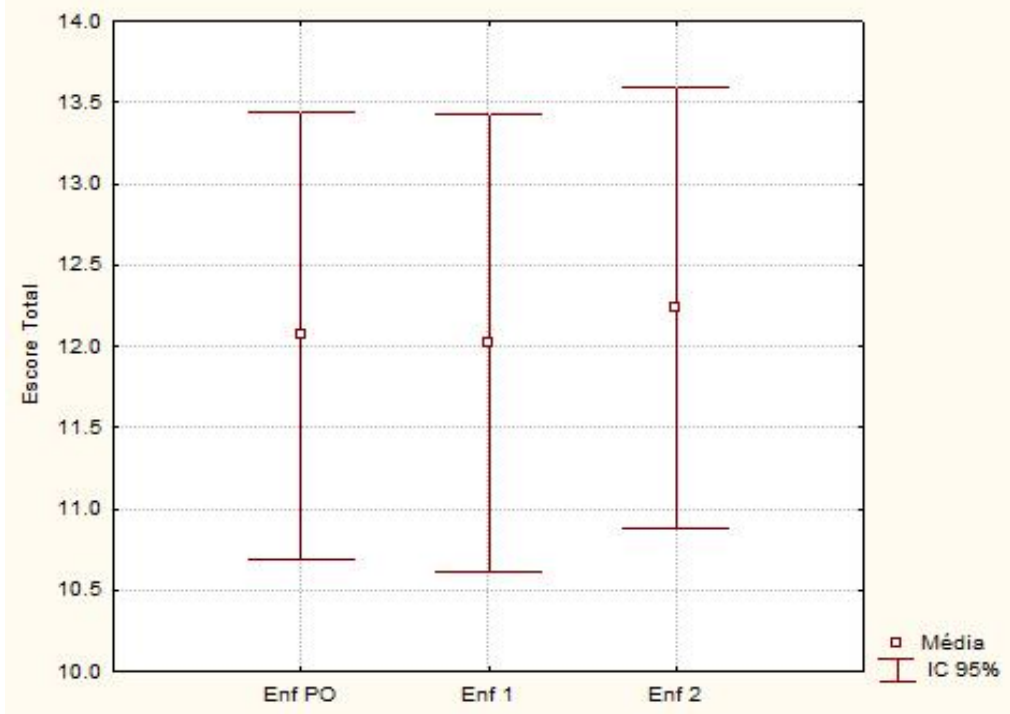

Legenda: Enf PO = enfermeira de referência para observação considerada padrão-ouro; Enf 1 = enfermeira clínica 1; Enf 2 = enfermeira clínica 2; Kappa Enf PO x Enf 1 = 0,738 - $p<0,001$; Kappa Enf PO x Enf $2=0,605-p<0,001$.

A Tabela 1 mostra a análise do coeficiente de correlação intraclasse (CCl) para o escore total e os subscores da PUSH na avaliação da cicatrização de úlceras venosas. Para o escore total, ambos os CCl foram acima de 0,9 indicando que a escala PUSH apresenta excelente confiabilidade interobservadores ${ }^{(13)}$ quando aplicada na avaliação da cicatrização de úlceras venosas.

Tabela 1: Coeficiente de correlação intraclasse $(\mathrm{CCl})$ entre os escores e subescores atribuídos pelo enfermeiro de referência para a observação tomada como padrão ouro (Enf PO) em comparação com os enfermeiros clínicos 1 e 2 (Enf 1 e Enf 2). Goiânia, GO, Brasil, 2017.

\begin{tabular}{|c|c|c|c|}
\hline Parâmetros da PUSH & $\mathrm{CCl}^{1}$ & $\mathbf{F}$ & $\mathbf{P}^{2}$-valor \\
\hline \multicolumn{4}{|l|}{ Área da ferida } \\
\hline Enf PO vs. Enf 1 & 0,997 & 607,42 & $<0,001$ \\
\hline Enf PO vs Enf 2 & 0,993 & 301,05 & $<0,001$ \\
\hline \multicolumn{4}{|l|}{ Quantidade de exsudato } \\
\hline Enf PO vs. Enf 1 & 0,927 & 25,90 & $<0,001$ \\
\hline Enf PO vs Enf 2 & 0,881 & 16,19 & $<0,001$ \\
\hline \multicolumn{4}{|l|}{ Tipo de tecido } \\
\hline Enf PO vs. Enf 1 & 0,672 & 5,25 & $<0,001$ \\
\hline Enf PO vs Enf 2 & 0,754 & 7,00 & $<0,001$ \\
\hline \multicolumn{4}{|l|}{ Escore total } \\
\hline Enf PO vs. Enf 1 & 0,992 & 246,82 & $<0,001$ \\
\hline Enf PO vs Enf 2 & 0,985 & 132,18 & $<0,001$ \\
\hline
\end{tabular}

Legenda: ${ }^{1}$ Coeficiente de correlação intraclasse; ${ }^{2} p \leq 0,05$.

\section{DISCUSSÃO}

As propriedades psicométricas da PUSH relacionadas à sua utilização por mais de um observador na avaliação da cicatrização de úlceras venosas foram objeto da presente investigação. Foram encontrados valores de índice Kappa compatíveis com confiabilidade interobservadores considerada como boa ou muito boa e coeficiente de correlação 
intraclasse com escores que variaram de moderada a muito boa correlação, indicando confiabilidade satisfatória quando aplicada para avaliação de úlceras venosas.

A confiabilidade interobservadores para uso da PUSH fora do seu contexto original (avaliação de lesão por pressão) já havia sido estudada, no entanto, apenas para pessoas com úlceras de perna, entre as quais 36\% eram úlceras venosas $^{(8)}$. O presente estudo, portanto, é o primeiro que avalia a confiabilidade interobservadores da PUSH exclusivamente para avaliação de úlceras venosas.

Este resultado assume especial relevância por criar evidências para uso da PUSH em estudos transversais, de caracterização de perfil de condições de cicatrização, e para mapear as características de úlceras em tratamento em determinado cenário.

A PUSH vem sendo aplicada para avaliar a evolução de cicatrização de lesões de diversas etiologias ${ }^{(6)}$, incluindo as úlceras de perna ${ }^{(8,18)}$, e as úlceras venosas ${ }^{(9,19-20)}$. Sua aplicação, nesse contexto, é recomendada com intervalos de, no mínimo duas semanas ${ }^{(8)}$.

Embora escala tenha se mostrado confiável quando aplicada por diferentes observadores, antes de ser adotada para uso na prática clínica, ainda deve ser avaliada quanto a sua consistência interna e responsividade na evolução da cicatrização de úlcera venosa, pois as características macroscópicas destas feridas são diferentes das úlceras arteriais, lesão por pressão, pé diabético ${ }^{(21)}$.

A PUSH apresenta como pontuação máxima o escore 10 para lesões com mais de $24 \mathrm{~cm}^{2}$, mas a área das úlceras venosas varia substancialmente, como pode ser visto, nessa pesquisa, foram encontrados valores desde $0,02 \mathrm{~cm}^{2} \mathrm{e}$ $551,0 \mathrm{~cm}^{2}$. Apesar de observado em outros países, variação na média das dimensões das lesões, como $29,3 \mathrm{~cm}{ }^{2(22)} \mathrm{e}$ $51,04 \mathrm{~cm}^{2(23)}$, os estudos brasileiros, apresentam lesões mais extensas, como de $50 \mathrm{~cm}^{2(9)}, 100 \mathrm{~cm}^{2}$ e maior que 160 $\mathrm{cm}^{2(24)}$.

Isso aponta para a necessidade de se estudar a responsividade da escala para avaliar a evolução da cicatrização de úlceras venosas, em estudos futuros, de modo a estabelecerem-se melhor suas propriedades psicométricas para aplicação fora do seu objetivo original.

Além disso, a PUSH considera na avaliação do tecido apenas a presença daquele que indica pior condição, e, por sua vez, as úlceras venosas geralmente apresentam esfacelo e granulação concomitantes, em diferentes proporções ${ }^{(20)}$, o que reforma a necessidade de estudar-se a sua responsividade.

A ausência de parâmetros do NPUAP para avaliação de exsudato impôs a necessidade de uma padronização para a presente pesquisa, que pode ser diferente daquela adotada dos demais estudos ${ }^{(6,8-9)}$ que omitiram, nos procedimentos, os critérios para essa avaliação. Espera-se que os próximos estudos possam descrever claramente o método de avaliação adotado, para que haja segurança em comparações futuras.

A classificação adotada na presente investigação parece ter sido apropriada para favorecer o julgamento clínico dos observadores, na correspondência entre os parâmetros avaliados e o escore da PUSH, uma vez que este item mostrou confiabilidade boa ou muito boa.

O item com confiabilidade interobservadores que teve menor escore, ainda que considerado bom, e com $\mathrm{CCl}$ moderada foi o tipo de tecido no leito da úlcera venosa. O tipo de tecidos classicamente descritos inclui epitelização, granulação, esfacelo, necrose ou escara ${ }^{(12,21)}$. Com a experiência clínica, percebe-se que o tecido de granulação pode variar entre vermelho escuro, vermelho vivo, vermelho tijolo e vermelho descorado, e ainda, que a necrose pode assumir aspecto de esfacelo marrom, amolecido, endurecido, amarelo firmemente aderido, amarelo frouxamente aderido, amarelo de aspecto mucinoso ${ }^{(24-25)}$ e diferentes densidades, podendo se apresentar como uma fina renda ou 
mosaico recobrindo o tecido de granulação, demandado julgamento criterioso e discriminação por parte do profissional que utiliza a PUSH, para a determinação do tipo de tecido (pior tecido) presente no leito da lesão.

Ademais, o uso da expressão "tipo de tecido no leito da lesão" pode levar o profissional a atentar-se menos para as pequenas escaras que podem estar presentes nas bordas. O prurido não é incomum e pode provocar coçadura que resulta em lesão por atrito no local.

Dessa forma, apesar de aparentemente simples, a PUSH suscita treino e calibração dos observadores, para sua utilização, assim como qualquer outro instrumento. Assim, é preciso considerar que o treinamento dos sujeitos realizado no presente estudo pode ter contribuído para o índice de concordância aqui obtido. Para uso em pesquisas ou na prática clínica, esse treinamento deve ser criteriosamente desenvolvido.

Como limitação do estudo aponta-se a amostra pequena de participantes com úlcera venosa. Contudo, destacase que a heterogeneidade das suas características clínicas pode ter contribuído para a expressão da variedade das condições que os observadores são desafiados e julgar na aplicação da PUSH nessa população.

\section{CONCLUSÃO}

A Pressure Ulcer Scale for Healing (PUSH) mostrou satisfatória confiabilidade interobservadores quando aplicada para avaliação de úlceras venosas, em um contexto com utilização de treinamento que precedeu a sua utilização. Esse treinamento implicou no acréscimo de instruções para avaliação e julgamento do exsudato e para medida da área da lesão.

\section{Apoio}

Conselho Nacional de Desenvolvimento Científico e Tecnológico (CNPq).

\section{REFERÊNCIAS}

1. Rüttermann M, Maier-Hasselmann A, Nink-Grebe B, Burckhardt M. Local treatment of chronic wounds: in patients with peripheral vascular disease, chronic venous insufficiency, and diabetes. Dtsch Arztebl Int [Internet]. 2013 [acesso em: 19 dez. 2018];110(3):25-31. Disponível em: https://doi.org/10.3238/arztebl.2013.0025.

2. Restrepo-Medrano JC. Instrumentos de monitorización clínica y medida de la cicatrización en úlceras por presión (UPP) y úlceras de la extremidad inferior (UEI). Desarrollo y validación de un índice de medida [Tese na Internet]. Alicante: Departamento de Enfermería Comunitaria, Medicina Preventiva y Salud Pública e Historia de la Ciencia de la Universidad de Alicante; 2010 [acesso em: 19 dez. 2018]. Disponível em: https://gneaupp.info/instrumentos-de-monitorizacion-clinica-y-medida-de-la-cicatrizacion-enulceras-por-presion-y-ulceras-de-la-extremidad-inferior-desarrollo-y-validacion-de-un-indice-de-medida/.

3. The National Pressure Ulcer Advisory Panel. PUSH Tool 3.0 (web version) | The National Pressure Ulcer Advisory Panel - NPUAP [Internet]. Washington, DC: The National Pressure Ulcer Advisory Panel [acesso em: 19 dez. 2018]. Disponível em: http://www.npuap.org/resources/educational-and-clinical-resources/push-tool/push-tool/.

4. Santos VLCG, Azevedo MAJ, Silva TS, Carvalho VMJ, Carvalho VF. Adaptação transcultural do pressure ulcer scale for healing (PUSH) para a língua portuguesa. Rev Lat Am Enfermagem [Internet]. 2005 [acesso em: 19 dez. 2018];13(3):305-13. Disponível em: https://doi.org/10.1590/S0104-11692005000300004.

5. Hon J, Lagden K, McLaren AM, O'Sullivan D, Orr L, Houghton PE, et al. A prospective, multicenter study to validate use of the PUSH in patients with diabetic, venous, and pressure ulcers. Ostomy Wound Manage [Internet]. 2010 [acesso em: 19 dez.

2018];56(2):26-36. Disponível em: https://www.o-wm.com/content/a-prospective-multicenter-study-validate-use-push@-patientswith-diabetic-venous-and-pressur.

6. Choi EP, Chin WY, Wan EY, Lam CL. Evaluation of the internal and external responsiveness of the Pressure Ulcer Scale for Healing (PUSH) tool for assessing acute and chronic wounds. J Adv Nurs [Internet]. 2016 [acesso em: 19 dez. 2018];72(5):1134-43.

Disponível em: https://doi.org/10.1111/jan.12898. 
7. Stotts NA, Rodeheaver GT, Thomas DR, Frantz RA, Bartolucci AA, Sussman C, et al. An instrument to measure healing in pressure ulcers: development and validation of the pressure ulcer scale for healing (PUSH). J Gerontol A Biol Sci Med Sci. 2001

Dec;56(12):M795-9.

8. Santos VLCG, Sellmer D, Massulo MME. Inter rater reliability of Pressure Ulcer Scale for Healing (PUSH) in patients with chronic leg ulcers. Rev Lat Am Enfermagem [Internet]. 2007 [acesso em: 19 dez. 2018];15(3):391-6. Disponível em: https://doi.org/10.1590/S0104-11692007000300005.

9. Santo PFE, Almeida SA, Silveira MM, Salomé GM, Ferreira LM. Use of the Pressure Ulcer Scale for Healing tool to evaluate the healing of chronic leg ulcers. Rev. Bras. Cir. Plást. [Internet]. 2013 [acesso em: 19 dez. 2018];28(1):133-41. Disponível em: https://doi.org/10.1590/S1983-51752013000100023.

10. Resende NM, Nascimento TC, Lopes FRF, Prates Júnior AG, Souza NM. Cuidado de pessoas com feridas crônicas na Atenção Primária à Saúde. Journal of Management \& Primary Health Care [Internet]. 2017 [acesso em: 19 dez. 2018];8(1):99-108. Disponível em: http://www.jmphc.com.br/jmphc/article/view/271.

11. Sibbald RG, Goodman L, Woo KY, Krasner DL, Smart H, Tariq G, et al. Special considerations in wound bed preparation 2011: an update@. Adv Skin Wound Care [Internet]. 2011 [acesso em: 19 dez. 2018];24(9):415-36. Disponível em:

https://doi.org/10.1097/01.ASW.0000405216.27050.97.

12. European Wound Management Association (EWMA). Position Document: Wound Bed Preparation in Practice. London: MEP Ltd, 2004.

13. Koo TK, Li MY. A Guideline of Selecting and Reporting Intraclass Correlation Coefficients for Reliability Research. J Chiropr Med [Internet]. 2016 [acesso em: 19 dez. 2018];15(2):155-63. Disponível em: https://doi.org/10.1016/j.jcm.2016.02.012.

14. Sardina D. Skin and Wound Resource Manual. Lake Geneva WI: Wound Care Education Institute; 2006.

15. Thomas DR, Rodeheaver GT, Bartolucci AA, Franz RA, Sussman C, Ferrell BA, et al. Pressure ulcer scale for healing: derivation and validation of the PUSH tool. The PUSH Task Force. Adv Wound Care. 1997;10(5):96-101.

16. Prefeitura Municipal de Belo Horizonte, Secretaria de Políticas Sociais, Secretaria Municipal de Saúde, Gerência de Assistência Coordenação de Atenção a Saúde do Adulto e do Idoso. Protocolo de assistência aos portadores de ferida. Revisão 2016 [Internet]. Belo Horizonte: Prefeitura Municipal de Belo Horizonte; 2006 [acesso em: 19 dez. 2018]. Disponível em:

http://www.pbh.gov.br/smsa/biblioteca/protocolos/curativos.pdf.

17. Kwiecien R, Kopp-Schneider A, Blettner M. Concordance analysis: part 16 of a series on evaluation of scientific publications. Dtsch Arztebl Int [Internet]. 2011 [acesso em: 19 dez. 2018];108(30):515-21. Disponível em:

https://doi.org/10.3238/arztebl.2011.0515.

18. Almeida SA, Moreira CNO, Salome GM. Pressure Ulcer Scale for Healing in monitoring of wound healing in elderly patients with leg ulcer. Rev. Bras. Cir. Plást. [Internet]. 2014 [acesso em: 19 dez. 2018];29(1):120-7. Disponível em: https://doi.org/10.5935/21771235.2014RBCP0020.

19. Medeiros ABA, Frazão CMQ, Tinôco JDS, Paiva MGMN, Lopes MVO, Lira ALBC. Venous ulcer: risk factors and the Nursing Outcomes Classification. Invest Educ Enferm [Internet]. 2014 [acesso em: 19 dez. 2018];32(2):252-9. Disponível em:

https://doi.org/10.17533/udea.iee.v32n2a08.

20. Malaquias SG, Bachion MM, Sant'Ana SMSC, Dallarmi CCB, Lino Junior RS, Ferreira PS. Pessoas com úlceras vasculogênicas em atendimento ambulatorial de enfermagem: estudo das variáveis clínicas e sociodemográficas. Rev Esc Enferm USP [Internet]. 2012 [acesso em: 19 dez. 2018];46(2):302-10. Disponível em: https://doi.org/10.1590/s0080-62342012000200006.

21. Dealey C. Cuidando de feridas: um guia para as enfermeiras. 3a ed. São Paulo: Atheneu; 2008.

22. Milic DJ, Zivic SS, Bogdanovic DC, Karanovic ND, Golubovic ZV. Risk factors related to the failure of venous leg ulcers to heal with compression treatment. J Vasc Surg [Internet]. 2009 [acesso em: 19 dez. 2018];49(5):1242-7. Disponível em:

https://doi.org/10.1016/i.jvs.2008.11.069.

23. Tuttle MS, Mostow E, Mukherjee P, Hu FZ, Melton-Kreft R, Ehrlich GD, et al. Characterization of bacterial communities in venous insufficiency wounds by use of conventional culture and molecular diagnostic methods. J Clin Microbiol [Internet]. 2011 [acesso em: 19 dez. 2018];49(11):3812-9. Disponível em: https://doi.org/10.1128/JCM.00847-11.

24. Nunes CAB. Evolução de úlceras vasculares em pessoas tratadas na atenção primária: abordagem sociodemográfica, clínica e terapêutica [dissertação]. [Goiânia]: Programa de Pós-Graduação em Enfermagem/UFG; 2012.

25. Oliveira BGRB, Nogueira GA, Carvalho MR, Abreu AM. Caracterização dos pacientes com úlcera venosa acompanhados no Ambulatório de Reparo de Feridas. Rev. Eletr. Enf. [Internet]. 2012 [acesso em: 19 dez. 2018];14(1):156-63. Disponível em: https://doi.org/10.5216/ree.v14i1.10322. 
APÊNDICE $1^{1}$

INSTRUMENTO DE APOIO PARA AVALIAÇÃO DE ÚLCERAS VENOSAS POR MEIO DA PRESSURE ULCER SCALE HEALING (PUSH)

I-Dados de identificação

Data: Cidade: Unidade:

Nome do participante:

\section{II- Condições da lesão}

-Localização da úlcera avaliada : MIE () MID( ) Zonas: 1 () 2() 3 ()

Plano: ( ) Anterior ( ) Posterior ( ) Lateral ( ) Medial ( ) Outro

\section{- Quantidade de exsudato:}

\begin{tabular}{|l|l|l|l|l|}
\hline Extensão de Impregnação & Grau de saturação da cobertura & Tempo de permanência & Tipo de cobertura & Julgamento \\
\hline ( ) < que a lesão & ( ) úmida & () $24 \mathrm{~h}$ & coxim & () ausente \\
( ) igual & () molhada & () $48 \mathrm{~h}$ & gazes & () pequeno \\
( ) um pouco maior & () saturada & () $72 \mathrm{~h}$ & espuma & () moderado \\
() muito maior & & () $>72 \mathrm{~h}$ & Bota Unna & () grande \\
& & & Outro & \\
\hline
\end{tabular}

- Mensuração: comprimento largura: área: $\mathrm{cm}^{2}$

\section{III - Aplicação da PUSH(3)}

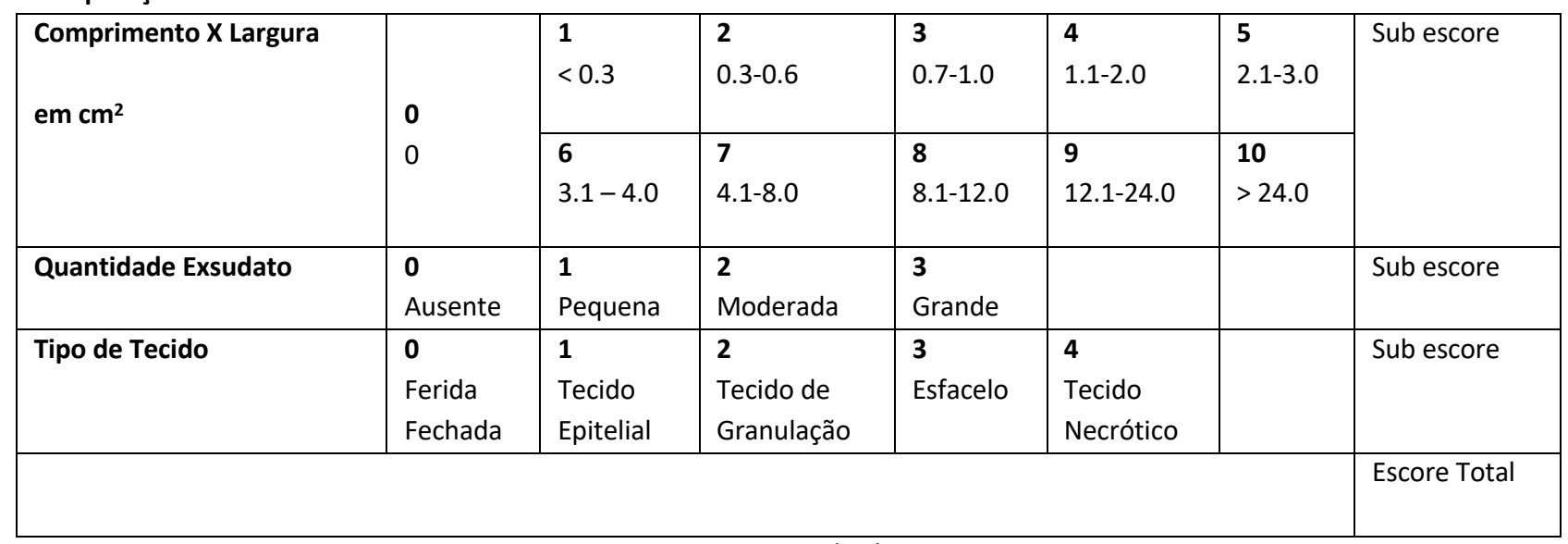

"Usado com permissão do National Pressure Ulcer Advisory Panel (01/03/2016)"

\footnotetext{
${ }^{1}$ Fonte: Alves GR. Confiabilidade e Responsividade da Pressure Ulcer Scale for Healing (PUSH) na avaliação de úlceras venosas [dissertation]. Goiânia; Programa de Pós-Graduação em Enfermagem/UFG; 2017.
} 